\title{
Viral Hepatitis in Patients Infected With Human Immunodeficiency Virus
}

\author{
Maria Helena P. Pavan, Francisco Hideo Aoki, \\ Dinaida Teresa Monteiro, Neiva Sellan L. Gonçales, \\ Cecília Amélia F. Escanhoela and Fernando L. Gonçales Júnior
}

\author{
Faculty of Medecine, UNICAMP; Hematology \\ and Hemoterapy Centre of UNICAMP, São \\ Paulo, Campinas, Brazil
}

\begin{abstract}
From 1992 to 1995 we studied 232 (69\% male, 87\% Caucasian) anti-human immunodeficiency virus (anti-HIV) positive Brazilian patients, through a questionnaire; HIV had been acquired sexually by $50 \%$, from blood by $32 \%$, sexually and/or from blood by $16.4 \%$ and by an unknown route by $1.7 \%$. Intravenous drug use was reported by $29 \%$; it was the most important risk factor for HIV transmission. The alanine aminotransferase quotient (qALT) was $>1$ for $40 \%$ of the patients, $93.6 \%$ had anti-hepatitis A virus antibody, $5.3 \%$ presented hepatitis $B$ surface antigen, $44 \%$ were anti-hepatitis $B$ core antigen positive and $53.8 \%$ were anti-hepatitis $\mathrm{C}$ virus (anti-HCV) positive. The anti-HCV test showed a significant association with qALT $>1$. Patients for whom the probable HIV transmission route was blood had a 10.8 times greater risk of being anti-HCV positive than patients infected by other routes. Among 30 patients submitted to liver biopsy, 18 presented chronic hepatitis.

Key Words: Hepatitis $\mathrm{C}$ virus, human immunodeficiency virus, alanine aminotransferase quotient, anti-hepatitis A antibody, hepatitis B surface antigen, anti-hepatitis B core antigen (anti-HBc).
\end{abstract}

Acquired immunodeficiency syndrome (AIDS) is characterized by a profound defect in cell-mediated immunity [1], which results in opportunistic infections and neoplasms that frequently involve the liver [2]. Risk factors and forms of transmission for human immunodeficiency virus (HIV) and hepatotropic viruses are often the same, which explains the high frequency of co-infection involving these two agents.

Hepatitis A virus (HAV) infection is common in both homosexual men [3] and intravenous drug users (IVDU) [4]. Like HIV, hepatitis B virus (HBV), hepatitis C virus (HCV) and hepatitis delta virus (HDV) can be spread both by needles and sexually [5].

Received on 28 August 2002; revised 10 March 2003. Address for correspondence: Dr. Maria Helena P. Pavan. Grupo de Estudos das Hepatites Virais do Hospital das Clínicas da UNICAMP. Av. Vital Brasil, 250. Campinas, SP, Brasil, Zip code: 13084-970. Email: jpavan@mpc.com.br

The Brazilian Journal of Infectious Diseases 2003;7(4):253-261 (C) 2003 by The Brazilian Journal of Infectious Diseases and Contexto Publishing. All rights reserved.
The prevalence of HCV infection among HIVseropositive subjects ranges from $4 \%$ to $67 \%$ [5], and although the sexual transmission of $\mathrm{HCV}$ is uncommon it can occur in patients co-infected with HIV [6-7], although, unlike HBV and HIV, HCV is not easily transmitted by homosexual intercourse [8]. Some studies have indicated that HIV infection accelerates the natural course of $\mathrm{HCV}$ infection and causes rapid progression to cirrhosis [9].

We investigated the etiologic agent, risk factors, clinical features, biochemical and immunological test results, liver biopsies and histopathological lesions of viral hepatitis in HIV-infected patients in order better understand the interaction between these two viral agents in Brazilian patients.

\section{Materials and Methods}

The study group consisted of 232 Brazilian patients from the city of Campinas (São Paulo state, Brazil) who had been assessed as positive at least twice in anti-HIV EIA-2 tests between 1992 and 1995. More 
than $2 / 3(69.4 \%)$ were men and $30.6 \%$ were women, with ages ranging from 17 to 63 years (mean $=30.8$ years); $87 \%$ were Caucasian and $13 \%$ were AfroBrazilian. According to the Center for Disease Control (CDC) criteria, $49.5 \%$ of the patients belonged to Group II, 30.2\% to Group III and $20.3 \%$ to Group IV.

All patients were interviewed using a standard questionnaire designed to identify a series of parameters, including known risk factors for HIV infection, such as previous blood transfusion, intravenous drug use (IVDU), sexual habits, sexual intercourse with HIV-infected or HIV-suspected partners, tattoos, previous acupuncture, previous surgeries and use of intravenous medication. Symptoms related to viral hepatitis were also evaluated, e.g. fever, nausea, vomiting, previous jaundice, fat intolerance, diarrhea, hematemesis, alcohol abuse and contact with hepatotoxins. All group members also underwent a complete physical examination.

At the first clinical evaluation a hemogram was made, and the levels of alanine aminotransferase (ALT), aspartate aminotransferase (AST), alkaline phosphatase (AP), bilirubin, albumin and gammaglobulin were assessed. Prothrombin activity was also evaluated. ALT activity was determined in each serum sample by the COBAS-MIRA automated assay (ROCHE) and the results expressed as the ALT quotient (qALT), calculated by dividing the ALT level in the sample by the maximum normal value for the method. When the ALT value was elevated, qALT was always $>1$.

The serum samples were also assessed for immunoglobulin-G (IgG) and immunoglobulin-M (IgM) anti-HAV using the "HAVAB-EIA"/"HAVABM" kit; HBsAg was quantified using the Auszyme Monoclonal kit, anti-HBcAg with the Corzyme kit, anti-HBsAg with the Ausab kit (Abbott) and anti$\mathrm{HCV}$ with a second generation HCV EIA (All kits were from Abbott Laboratories, North Chicago, IL., USA).

Liver biopsy was performed on all patients $(n=30)$ with $\mathrm{qALT}>1.5$ times the normal value, or who presented hepatomegaly in the physical examination, or who were anti-HCV positive with elevated ALT, or HBsAg positive with elevated ALT or to investigate patients with fever of unknown origin. Diagnoses were: normal liver (NL), chronic persistent hepatitis $(\mathrm{CPH})$, chronic active hepatitis $(\mathrm{CAH})$, chronic lobular hepatitis $(\mathrm{CLH})$, minimal changes (MC), liver cirrhosis (LC) or other nonrelated lesions. CAH was classified as mild, moderate or intense. Hematoxylin-eosin, GrocottGomori, Masson's trichrome, Ziehl-Neelsen and Perls's Prussian staining was also used.

Statistical analysis. Chi-squared analyses (or Fisher's exact test when indicated) were used to compare the risk factors for HIV infection and serological tests for HCV and HBV. The EPI INFO program was used to calculate the results, with $\mathrm{p} \leq$ 0.05 being considered statistically significant. The relative odds ratio was calculated to compare the strength of the association of a positive anti-HCV result with the different risk factors. A logistic regression model (SAS program) was used to assess risk factors for qALT $>1$. All percentages were calculated using $n=232$ patients, unless otherwise indicated.

\section{Results}

The questionnaire completed by the 232 patients showed that the most likely HIV acquisition routes were as follows: sexual intercourse for $50 \%$ of the patients, from infected blood for $32 \%$, sexually and/ or from infected blood for $16.3 \%$ and by unknown routes for $1.7 \%$. In $29 \%$ of the patients IVDU was the most important risk factor found. Other risk factors were: heterosexual intercourse with an HIVinfected partner for $17 \%$, homosexual intercourse for $13.4 \%$, promiscuous heterosexual practices for $11 \%$, promiscuous heterosexual practices and IVDU for $7 \%$, male bisexuality for $5 \%$ and sexual intercourse with a person at risk of HIV infection for $3.4 \%$. Together these factors accounted for $86 \%$ of all cases. 
Table 1. Biochemical results for HIV infected patients (n)

\begin{tabular}{lrrrrrr}
\hline Biochemical tests & \multicolumn{2}{c}{ Abnormal $^{1}$} & \multicolumn{2}{c}{ Normal } & \multicolumn{2}{c}{ Total } \\
& $\mathbf{n}$ & $\boldsymbol{\%}$ & $\mathbf{n}$ & $\mathbf{\%}$ & $\mathbf{n}$ & $\%$ \\
\hline Alanine aminotransferase (ALT) & 92 & 40.0 & 140 & 60.0 & 232 & 100 \\
Aspartate aminotransferase (AST) & 49 & 21.0 & 182 & 79.0 & 231 & 100 \\
Alkaline phosphatase (AP) & 17 & 8.0 & 195 & 92.0 & 212 & 100 \\
Total bilirubin & 9 & 5.6 & 153 & 94.4 & 162 & 100 \\
Albumin & 10 & 5.0 & 92 & 95.0 & 202 & 100 \\
Gamma-globulin & 131 & 66.0 & 67 & 34.0 & 198 & 100 \\
\hline
\end{tabular}

(1)All values were above the upper normal value for the method, except for albumin which was below the lower normal value for the method.

Table 2. Serological tests for hepatitis virus A, B and C (HAV, HBV, HCV, respectively) in HIV-infected patients (n)

\begin{tabular}{lrrrrrrrr}
\hline Serologic tests & \multicolumn{2}{c}{ Positive } & \multicolumn{2}{c}{ Negative } & \multicolumn{2}{c}{ Indeterminate } & \multicolumn{2}{c}{ Total } \\
& n & \% & \multicolumn{1}{c}{ n } & \% & n & \% & n & \% \\
\hline IgM anti-HAV & 01 & 0.6 & 155 & 99.4 & - & - & 156 & 100 \\
IgG anti-HAV & 146 & 93.6 & 10 & 6.4 & - & - & 156 & 100 \\
HbsAg & 12 & 5.3 & 211 & 93.4 & 3 & 1.3 & 226 & 100 \\
Anti-HbcAg & 100 & 44.0 & 126 & 56.0 & - & - & 226 & 100 \\
Anti-HbsAg & 79 & 35.3 & 145 & 64.7 & - & - & 224 & 100 \\
Anti-HCV & 119 & 53.8 & 100 & 45.2 & 2 & 1.0 & 221 & 100 \\
\hline
\end{tabular}

Table 3. Positive serological tests for hepatitis virus B (HBV) and C (HCV) according to HIV transmission route

\begin{tabular}{lcccc}
\hline Positive serologic test & \multicolumn{2}{c}{ Blood transmission } & \multicolumn{2}{c}{ Sexual transmission } \\
& n & \% & n & \% \\
\hline Anti-HbcAg & 5 & 15.6 & 26 & 66.7 \\
Anti-HCV & 27 & 84.4 & 13 & 33.3 \\
Total (n) & 32 & 100.0 & 39 & 100.0 \\
\hline
\end{tabular}


Table 4. Probable risk factors involved in hepatic lesions based on the alanine aminotransferase quotient (qALT) of patients ( $n$ )

\begin{tabular}{|c|c|c|c|c|c|c|}
\hline \multirow[t]{2}{*}{ Risk Factor for hepatic lesion } & \multicolumn{2}{|c|}{ q A $\mathbf{A L T}>1$} & \multicolumn{2}{|c|}{ q $\mathbf{A L T} \leq 1$} & \multicolumn{2}{|c|}{ Total } \\
\hline & $\mathbf{n}$ & $\%$ & $\mathbf{n}$ & $\%$ & $\mathbf{n}$ & $\%$ \\
\hline No risk factors & 5 & 5.4 & 49 & 35.0 & 54 & 23.3 \\
\hline One risk factor & 42 & 45.6 & 50 & 35.7 & 92 & 39.6 \\
\hline HBV(HbsAg+) & 2 & 2.2 & - & - & 2 & 0.9 \\
\hline $\mathrm{HCV}($ anti-HCV +) & 27 & 29.3 & 27 & 19.3 & 54 & 23.3 \\
\hline Hepatotoxic drugs & 12 & 13.0 & 17 & 12.1 & 29 & 12.5 \\
\hline Use of illicit drugs ${ }^{1}$ & 1 & 1.1 & 1 & 0.7 & 2 & 0.9 \\
\hline Alcohol abuse & - & - & 5 & 3.6 & 5 & 2.1 \\
\hline Two risk factors & 29 & 31.5 & 22 & 15.7 & 51 & 22.0 \\
\hline $\mathrm{HBV}+\mathrm{HCV}$ & - & - & 1 & 0.7 & 1 & 0.4 \\
\hline HBV + other & - & - & 1 & 0.7 & 1 & 0.4 \\
\hline $\mathrm{HCV}+$ other & 29 & 31.5 & 20 & 14.3 & 49 & 21.1 \\
\hline Two or more non-viral risk factors & 2 & 2.2 & 5 & 3.6 & 7 & 3.0 \\
\hline $\mathrm{HBV}+\mathrm{HCV}+$ other $^{2}$ & 4 & 4.4 & 3 & 2.1 & 7 & 3.0 \\
\hline All factors & 1 & 1.1 & - & - & 1 & 0.4 \\
\hline Serological tests not made & 9 & 9.8 & 11 & 7.8 & 20 & 8.6 \\
\hline Total & 92 & 100.0 & 140 & 100.0 & 232 & 100.0 \\
\hline
\end{tabular}

(1)Illicit drug = cannabis or cocaine (as snuff or intravenously).

(2) Other $=$ hepatotoxic drug, illicit drug or alcohol abuse.

Table 5. Liver biopsy results for HIV positive patients (n).

\begin{tabular}{lrr}
\hline Histopathological Features & n & \% \\
\hline Chronic active hepatitis (CAH) & 17 & 56.7 \\
Chronic persistent hepatitis (CPH) & 1 & 3.3 \\
Cholestasis & 3 & 10.0 \\
Liver carcinoma & 1 & 3.3 \\
Minimal changes & 6 & 20.0 \\
Tuberculosis & 1 & 3.3 \\
Normal liver & 1 & 3.3 \\
Total & 30 & 100.0 \\
\hline
\end{tabular}


Other factors that were associated with HIV and viral hepatitis were: use of non-disposable needles $(55.6 \%)$, previous surgery $(55.6 \%)$; tattoos $(31.5 \%)$, blood transfusion (14.6\%) and acupuncture (2.2\%). Daily alcohol consumption was reported by $14 \%$. Abuse of non-alcohol drugs was cited by $13 \%$ of the patients, 13 of 30 of whom were intravenous users, while 23 of 30 used inhalation as the administration route. Hepatotoxic drug use was reported by $33.2 \%$. However, there was no significant association between these factors and qALT $>1(\mathrm{p}>0.05)$. Prior hepatitis was reported by $15 \%$ of the patients, but the etiological agents were unknown.

We found that $28.4 \%$ of the patients had some symptoms of digestive tract disorder, nausea $(23.7 \%)$ being the most frequently reported symptom, while $10.3 \%$ complained of diarrhea and $9.5 \%$ complained of vomiting. Jaundice was cited by $4.3 \%$ of the patients, but only two complained of hematemesis. Only $3.5 \%$ presented moderate to intense grade hepatomegaly (liver $>3 \mathrm{~cm}$ ), while in $54.3 \%$ the liver was $0-3 \mathrm{~cm}$ from the right costal margin and in $42.2 \%$ the liver was not palpable. The presence of a palpable liver was significantly correlated with qALT $>1(\mathrm{p}=0.0001)$. The spleen was palpable in $12.5 \%$, jaundice was found in $1.3 \%$, $37.5 \%$ had cervical adenomegaly and one patient had the clinical features of portal hypertension.

Table 1 shows the results of the biochemical tests. Of the 49 patients with elevated AST levels, 22 used some kind of hepatotoxic medication, while among the 182 patients with normal AST, 55 (30\%) also used hepatotoxic medication; patients who used hepatotoxic medication were significantly more likely to have high AST levels. Of the 17 patients with elevated AP levels, three had tuberculosis. Ten subjects with elevated AP levels were submitted to abdominal ultrasound but neither adenomegaly nor cholestasis were found. Among the 10 patients with hypoalbuminemia, five were classified in group IV of the CDC classification.

Table 2 shows the results of the serological tests for HAV, HBV and HCV. Of the 156 patients tested, 93.6\% were anti-HAV IgG positive while one patient was anti-HAV IgM positive. Among the 226 patients, $5.3 \%$ were $\mathrm{HBsAg}$ positive and $44.2 \%$ anti-HBcAg positive. Seventy-nine of 224 (35.3\%) patients were anti-HBsAg positive and 119 of 221 $(53.8 \%)$ of the patients were anti-HCV positive. Table 3 shows the distribution of only anti-HBcAg or only anti-HCV positive patients, with respect to HIV transmission route. There was a significant association $(p=0.001)$ between these factors, and we observed that patients whose probable HIV transmission route was by blood had a 10.8 times greater risk of being anti-HCV positive than those whose probable HIV transmission route was sexual intercourse.

Table 4 shows the probable risk factors involved in hepatic lesions based on qALT values. Among 92 patients with qALT $>1,4 \%$ had no detectable hepatic lesions, although one presented cervical adenomegaly that later was diagnosed as ganglionic tuberculosis and one had syphilis (VDRL=1/128). Table 4 also shows that $45.6 \%$ of the 92 patients with qALT $>1$ had only one casual risk factor for hepatic lesion, i.e. HBV or HCV infection, use of hepatotoxic or illicit drugs or alcohol abuse. One of the 92 patients had all the risk factors that we investigated. If we consider all the individuals with qALT $>1$ and that presented a viral hepatitis marker, we see that $66.3 \%$ were positive for anti-HCV and four (4\%) were co-infected with HBV and HCV. The factor that gave a significant association with patients who had a qALT $>1$ was a positive antiHCV test $(p=0.0001)$.

Thirty patients agreed to have a liver biopsy (table 5); 18 presented chronic hepatitis and one had a normal liver.

\section{Discussion}

In $50 \%$ of our patients, sexual intercourse was the principal risk factor for HIV infection. This route of transmission is associated with about half of the cases of AIDS in adults in Brazil [10-11]. HIV transmission by blood probably occurred in $32 \%$ of our patients, with $29 \%$ being associated with IVDU. Official figures 
for the state of São Paulo [11] show that 35\% of the AIDS cases in this region are related to IVDU, which indicates that the population studied by us was representative of the HIV-infected adult population in our part of Brazil.

Neither alcoholism nor IVDU was significantly associated with high ALT levels in our group of patients, although elevated ALT levels did significantly correlate with HCV infection, and hepatomegaly and high AST levels were associated with hepatotoxicity. We consider that all HIVpositive patients with elevated ALT levels are at risk of HCV infection and should be investigated. A large number of AIDS patients are at present undergoing potentially hepatotoxic highly active antiretroviral therapy (HAART) therapy, and these patients must have their AST levels periodically measured.

About $28 \%$ of the patients in our study complained of gastrointestinal symptoms, nausea and vomiting being the most common (about 33\%) with previous jaundice $(4.3 \%)$ and hematemesis (1\%) being uncommon, while at clinical evaluation only $1.3 \%$ were diagnosed as having jaundice and about $0.5 \%$ had portal hypertension. In our clinical experience, about $94 \%$ of the anti-HCV positive blood donors who are anti-HIV negative have no symptoms of jaundice, and only $2 \%$ report previous jaundice [12]. In the group of HIVpositive patients investigated in our current 1study, there were usually no symptoms of viral hepatitis (a few patients showed mild symptoms), just as was the case in HIV-negative patients with chronic viral hepatitis C seen in our general practice.

In general, HIV and HCV positive patients have a higher frequency of hepatomegaly when compared with patients infected only with HCV [2]. This may be explained by the presence of another hepatotropic virus, opportunistic infections, or the use of medication. Elevated ALT levels are frequently observed in HIVinfected subjects and may also be associated with hepatotoxic drugs, bad nutrition, cardiac insufficiency, hypoxia, medical procedures and blood transfusions. The majority of these factors were absent from our group of patients with elevated ALT levels.

In $17(8 \%)$ of our patients, AP levels were elevated, probably due to intrahepatic cholestasis or hilar adenomegaly (each of which may be present in cases of tuberculosis), tuberculosis being detected in three of 17 of these patients. In our opinion all HIV-infected patients with elevated AP levels should be submitted to liver biopsy, especially if they have fever.

Hypoalbuminemia was present in $5 \%$ of our patients, this being common in AIDS patients, due to bad nutrition, and $50 \%$ of our patients had opportunistic infections. It appears that both AIDS and opportunistic infections were important factors in patients' hypoalbuminemia, which was itself made worse by concomitant HBV or HCV infections. Elevated gamma-globulin levels were very frequently observed in our group of AIDS patients, but it was not associated with advanced liver disease.

About $94 \%$ of our patients had anti-HAV IgG in their serum. There is a higher risk of HAV infection among homosexual men [3] and IVDU [13], both of these being high risk groups for HIV infection. A high prevalence of anti-HAV has also been observed among HIV-negative adults in developing and newly industrialized countries, such as Brazil [14].

There were 12 (5.3\%) HbsAg-positive patients in our group, this being about three times higher than the frequency found in a group of blood donors previously studied by us [15]. Six $(50 \%)$ of our HbsAg-positive patients were IVDU, two (16.7\%) were homosexual males, one $(8 \%)$ was a bisexual male, one $(8 \%)$ was a promiscuous heterosexual and two (17\%) presented more than one risk factor. Different values have been found in other studies, e.g. in Spain 5\% of homosexual men were $\mathrm{HBsAg}$ positive, $13 \%$ were IVDU and $2 \%$ of the patients' sexual partners were also HBsAg positive [16]; these values were similar in both HIV-positive and negative patients.

About $44 \%$ of our HIV-positive patients were also anti-HBcAg positive. In the group of blood donors that we had studied earlier [15], 11\% were anti-HBcAg positive. In the current study there was no significant difference in the prevalence of anti$\mathrm{HBcAg}$ in IVDU (40\%) and homosexuals (44\%), 
but when the relationship between the presence of anti-HBcAg and mode of transmission was analyzed it was found that HIV-positive patients who had been contaminated by the sexual route had a significantly higher prevalence of anti-HBcAg than patients contaminated by blood. These results reflect the importance of the sexual transmission of HBV. In some patients anti-HBcAg may act as the only HBV infection for several reasons, e.g. undetectable levels of HBsAg in a serological test (the so-called immunological window), deficient production of antiHBsAg caused by genetic factors, loss of HBsAg in very old infections, passive transmittance of anti$\mathrm{HBcAg}$ by drug users, a false positive EIA test result, or interference caused by the presence of other viruses. In our study, 25 patients, $48 \%$ of whom were IVDU, presented anti-HBcAg as the only HBV marker. It appears that some patients co-infected with HIV are unable to produce anti-HBsAg during $\mathrm{HBV}$ infection. This atypical feature of HBV infection has been observed in $10.7 \%$ of IVDU in England and $35 \%$ in Italy [17].

The anti-HBsAg antibody was present in $35.3 \%$ of our patients, lower than the $65 \%$ found in patients with advanced HIV infection [18]. Another study [16] found anti-HBsAg in $88 \%$ of IVDU, $70 \%$ of homosexual men and $26 \%$ among sexual partners of IVDU. In our study, 15 patients presented only anti-HBsAg as a marker of previous HBV infection. These patients had not previously been immunized against HBV and it seems that they had lost the other anti-HBV markers due to HIV infection. No significant correlation was observed between the presence of HBV markers and elevated ALT levels. About $54 \%$ of our patients were anti-HCV positive, which is very much higher than the $2.6 \%$ anti-HCV positive found in blood donors from the region of Campinas [15]. When the probable route of HIV transmission was by blood we noted a higher rate of positive anti-HCV tests $(84.4 \%)$ when compared to that observed in patients whose probable HIV transmission route was sexual intercourse (33.3\%).

This correlation was significant and confirms that hepatitis $\mathrm{C}$ is very common when HIV infection is acquired by exposure to infected blood. The prevalence of positive anti-HCV tests was high among IVDU (90\%) and low among homosexual men $(3.7 \%)$, while among promiscuous heterosexuals there was a higher prevalence of HCV $(43.5 \%)$ than in homosexuals $(3.7 \%)$. A study involving the sexual partners of patients with hemophilia [7] found that the rate of $\mathrm{HCV}$ transmission was five times higher in the presence of concomitant HIV transmission; it may be that HIV is an important cofactor for the sexual transmission of HCV.

Some authors $[19,20,21]$ consider that anti-HCV tests can present false positives in patients with HIV infection due to the high levels of circulating antibodies, while others argue that anti-HCV tests can give false negative results in cases of advanced AIDS because of decreased antibody production [22]. Some studies have shown a good correlation between the results of anti-HCV tests by EIA-2 and tests involving HCV RNA using the polymerase chain reaction (PCR); one such study showed that in groups with high risk for $\mathrm{HCV}$ infection about $93 \%$ of the positive anti-HCV EIA-2 tests were positive for the immunoblot test for HCV (RIBA-2), and $85 \%$ of them were HCV RNA positive [23-24]. In our opinion, anti-HCV positive patients should always be considered as HCV infected, which may be confirmed by detecting HCV-RNA using PCR.

With regard to our findings concerning ALT, other authors have also observed an association between positive anti-HCV test and elevated plasma ALT [2024]. We consider that the cytopathic effect of HCV in the liver may be amplified when the patient is co-infected with HIV.

We encountered chronic hepatitis in $60 \%$ of the liver biopsies from HIV patients (only $3.3 \%$ had a normal liver) and $70 \%$ of our anti-HCV positive patients had $\mathrm{CAH}$, which demonstrates the importance of liver biopsy in co-infected patients. In the past a great number of opportunistic infections have been seen in liver biopsies of HIV-infected patients because such patients normally had advanced AIDS [25-26-27]. In more recent studies, patients without advanced AIDS have been 
evaluated, and we have observed histopathological lesions similar to those found in patients with $\mathrm{HCV}$ but without HIV. In such cases, changes such as $\mathrm{CAH}$ and $\mathrm{CH}$, which are associated with hepatotropic virus, predominate [28].

In HIV patients co-infected with HBV the liver biopsies showed mild lesions, when compared to the histopathological alterations present in anti-HIV negative patients [29]; it seems that HBV is not cytopathic for hepatocytes. The mild liver lesions observed in our HBV infected patients reflects a deficient cellular immune response to infected hepatocytes in patients with HIV infection. The occurrence of the more severe lesions, which were observed in HCV patients co-infected with HIV suggests, and perhaps confirms, the predominantly cytopathic action of HCV in the liver [9].

In conclusion, the occurrence of viral hepatitis in our group of Brazilian HIV-infected patients is common and it affects the clinical course of both infections. The advent of highly effective anti-HIV therapy has given AIDS patients both prolonged survival and a better quality of life, but an increased incidence of end-stage liver disease is now being observed among these patients and this will oblige us to find better ways to treat the chronic liver diseases caused by hepatotropic viruses.

\section{References}

1. Reichert C.M., O’leary T.J., Levens D.L., et al. Autopsy pathology in the acquired immunodeficiency syndrome. A J P 1983; 112(3):357-82.

2. Lebovics E., Thung S.N., Schaffner F., Radensky P.W. The liver in the acquired immunodeficiency syndrome: a clinical and histological study. Hepatology 1985;5(2):293-8.

3. Corey L.E., Holmes K.K. Sexual transmission of hepatitis a in homosexual men. N Engl J Med 1980;302:435-8.

4. MMWR 1988;37(19):297-305.

5. Horvath J., Raffanti S.P. Clinical aspects of the interactions between human immunodeficiency virus and the hepatotropic viruses. Clin Infect Dis 1994;18:339-47.

6. Lissen E., Sanchez-Quijano A., Leal M.et al. Sexual transmission of hcv and possible role of coexistent hiv infection in the index cases. J Hepatology 1991;13 (suppl.2):s44.
7. Eyster M.E., Alter H.H., Aledort L.M., et al Heterosexual co-transmission of hepatitis c virus (hcv) and human immunodeficiency virus (hiv). Ann Intern Med 1991; 115:764-8.

8. Melbye M., Biggar R.J., Wantzin P., et al. Sexual transmission of hepatitis c virus: cohort study (19811989) among european homosexual men. Br Med J 1990;301:210-2.

9. Zylberberg H., Pol S. Reciprocal interactions between human immunodeficiency virus and hepatitis $\mathrm{c}$ virus infection. Clin Infect Dis 1996;23:1117-25.

10. Boletim epidemiológico - Aids - Ministério da Saúde Coordenação Nacional de Doenças Sexualmente Transmissíveis e Aids. Semana epidemiológica 36 a 48 - setembro a novembro de 1997.

11. Boletim Epidemiológico - Programa DST/Aids - Secretaria de Estado da Saúde - s.p. - agosto de 1995.

12. Gonçales Jr., F.L. Avaliação clínico-epidemiológica prospectiva de candidatos a doadores de sangue antihcv reagentes. Campinas, 1998. Título de professor livre - docente. Unicamp.

13. Scheutz F., SkinhoJ. P., Mark I. Viral hepatitis among parenteral drug addicts attending a danish addiction clinic. Scand J Infect Dis 1983;15:139-43.

14. Pannuti C.S., Mendonça J.S., Carvalho M.J.M., et al. Hepatitis a antibodies in two socio-economically distinct populations of São Paulo, Brazil. Rev Inst Med Trop São Paulo 1985;27:162-4.

15. Gonçales Jr., F.L., Boccato R.B.S., Pedro R.J., et al. Prevalências do Hbsag, do anti-Hbc e do anti-Hcv na população de candidatos a doadores de sangue do hemocentroCampinas. Rev Inst Med Trop São Paulo 1993;35:45-51.

16. Tor J., Llibre J.M., Carbonell M., et al. Sexual transmission of hepatitis $\mathrm{c}$ and its relation with hepatitis $\mathrm{b}$ virus and hiv. Br Med J 1990;301:1130-3.

17. Levine O.S., Vlahov D., Nelson K.E. Epidemiology of hepatitis b virus infections among injecting drug users: seroprevalence, risk factors, and viral interactions. The Johns Hopkins University School of Hygiene and Public Health 1994; 16:418-36.

18. Homann C., Krogsgaard K., Pedersen C., et al. High incidence of hepatitis $\mathrm{b}$ infection and evolution of chronic hepatitis $b$ infection in patients with advanced hiv infection. Journal of acquired immune deficiency syndromes 1991;4:416-20.

19. McNair A.N.B., Janice M.R.C.P., Thomas H.C. Interactions of the human immunodeficiency virus and the hepatotropic viruses. Seminars in Liver Disease 1992;12(no.2):188-96.

20. Sherman K.E., Freeman S., Harrison S., Andron L. Prevalence of antibody to hepatitis c virus in patients infected with the human immunodeficiency virus. JID 1991; 163:414-5. 
21. Mchutchinson J.G., Polito A., Person J.L., et al. Assessment of hepatitis c antibody tests in homosexual men with hyperglobulinemia. J Infect Dis 1991; 164:217-8.

22. Chamot E., Hirschel B., Wintsch J.et al. Loss of antibodies against hepatitis $\mathrm{c}$ virus in hiv-seropositive intravenous drug users. Aids 1990;4(12):1275-7.

23. Gretch, d.r. - diagnostic tests for hepatitis c. Hepatology 1997;26(suppl)43s.

24. Quan C.M., Krajden M., Grigoriew G.A., Salit I.E. Hepatitis c virus infection in patients infected with the human immunodeficiency virus. Clin Infect Dis 1993;17:117-9.

25. Glasgow B.J., Anders K., Layfield L.J., et al. Clinical and pathologic findings of the liver in the acquired immune deficiency syndrome (Aids). Am J Clin Pathol 1985;83:582-8.

26. Kahn S.A., Saltzman B.R., Klein R.S.,et al. Hepatic disorders in the acquired immunodeficiency syndrome: A clinical and pathological study. Am J Gastroenterol 1986;81(12):1145-8.

27. Dworkin B.M., Stahl R.E., Giardina M.A., et al. The liver in acquired immune deficiency syndrome: emphasis on patients with intravenous drug abuse. Am J Gastroenterol 1987;82(3):231-6.

28. Reias J., Lopes I. Influence of hiv infection in chronic hepatitis $\mathrm{c}$ in intravenous drug addicts. Hepatology 1992;16:19a:602, 1992.

29. Goldin R.D., Fish D.E., Hay A., et al Histological and immunohistochemical study of hepatitis b virus in human immunodeficiency virus infection. J Clin Pathol 1990; $43: 203-5$. 